\section{Analysis of Attained Height and Diabetes Among 554,122 Adults Across 25 Low- and Middle- Income Countries}

Diabetes Care 2020;43:2403-2410 | https://doi.org/10.2337/dc20-0019

\section{OBJECTIVE}

The prevalence of type 2 diabetes is rising rapidly in low-income and middle-income countries (LMICs), but the factors driving this rapid increase are not well understood. Adult height, in particular shorter height, has been suggested to contribute to the pathophysiology and epidemiology of diabetes and may inform how adverse environmental conditions in early life affect diabetes risk. We therefore systematically analyzed the association of adult height and diabetes across LMICs, where such conditions are prominent.

\section{RESEARCH DESIGN AND METHODS}

We pooled individual-level data from nationally representative surveys in LMICs that included anthropometric measurements and diabetes biomarkers. We calculated odds ratios (ORs) for the relationship between attained adult height and diabetes using multilevel mixed-effects logistic regression models. We estimated ORs for the pooled sample, major world regions, and individual countries, in addition to stratifying all analyses by sex. We examined heterogeneity by individual-level characteristics.

\section{RESULTS}

Our sample included 554,122 individuals across 25 population-based surveys. Average height was $161.7 \mathrm{~cm}$ (95\% Cl 161.2-162.3), and the crude prevalence of diabetes was 7.5\% (95\% $\mathrm{Cl} 6.9-8.2)$. We found no relationship between adult height and diabetes across LMICs globally or in most world regions. When stratifying our sample by country and sex, we found an inverse association between adult height and diabetes in $5 \%$ of analyses ( 2 out of 50 ). Results were robust to alternative model specifications.

\section{CONCLUSIONS}

Adult height is not associated with diabetes across LMICs. Environmental factors in early life reflected in attained adult height likely differ from those predisposing individuals for diabetes.

Noncommunicable diseases (NCDs), such as type 2 diabetes, are rapidly rising in lowincome and middle-income countries (LMICs) $(1,2)$, and there is an urgent need to understand factors driving this rise. To explore knowledge gaps that remain unexplained by more established risk factors (3), increasing attention has been paid to early life factors in chronic disease etiology (4). The underlying Developmental Origins

\author{
Felix Teufel, ${ }^{1}$ Pascal Geldsetzer, ${ }^{1,2}$ \\ Jennifer Manne-Goehler, ${ }^{3,4}$ \\ Omar Karlsson, ${ }^{5,6}$ Viola Koncz, ${ }^{1,7}$ \\ Andreas Deckert, ${ }^{1}$ Michaela Theilmann, ${ }^{1}$ \\ Maja-Emilia Marcus, ${ }^{8}$ Cara Ebert, ${ }^{9}$ \\ Jacqueline A. Seiglie, ${ }^{10,11}$ \\ Kokou Agoudavi, ${ }^{12}$ \\ Glennis Andall-Brereton, ${ }^{13}$ \\ Gladwell Gathecha, ${ }^{14}$ Mongal S. Gurung, ${ }^{15}$ \\ David Guwatudde, ${ }^{16}$ Corine Houehanou, ${ }^{17}$ \\ Nahla Hwalla, ${ }^{18}$ Gibson B. Kagaruki, ${ }^{19}$ \\ Khem B. Karki, ${ }^{20}$ Demetre Labadarios, ${ }^{21}$ \\ Joao S. Martins, ${ }^{22}$ Mohamed Msaidie, ${ }^{23}$ \\ Bolormaa Norov, ${ }^{24}$ Abla M. Sibai, ${ }^{25}$ \\ Lela Sturua, ${ }^{26}$ Lindiwe Tsabedze, ${ }^{27}$ \\ Chea S. Wesseh, ${ }^{28}$ Justine Davies, ${ }^{29,30}$ \\ Rifat Atun, ${ }^{31,32}$ Sebastian Vollmer, ${ }^{8}$ \\ S.V. Subramanian, ${ }^{33,34}$ \\ Till Bärnighausen, 1,31,35 \\ Lindsay M. Jaacks, ${ }^{31,36}$ and \\ Jan-Walter De Neve
}

${ }^{1}$ Heidelberg Institute of Global Health, Faculty of Medicine and University Hospital, Heidelberg University, Heidelberg, Germany

${ }^{2}$ Division of Primary Care and Population Health, Department of Medicine, Stanford University, Stanford, CA ${ }^{3}$ Division of Infectious Diseases, Brigham and Women's Hospital, Boston, MA

${ }^{4}$ Medical Practice Evaluation Center, Massachusetts General Hospital, Boston, MA

${ }^{5}$ Takemi Program in International Health, Harvard T.H. Chan School of Public Health, Boston, MA ${ }^{6}$ Centre for Economic Demography, Lund University, Lund, Sweden

${ }^{7}$ Institute for Medical Information Processing, Biometry and Epidemiology, Ludwig-Maximilians University of Munich, Munich, Germany

${ }^{8}$ Department of Economics and Centre for Modern Indian Studies, Georg-August-Universität Göttingen, Göttingen, Germany

${ }^{9}$ RWI - Leibniz Institute for Economic Research, Essen (Berlin Office), Germany

${ }^{10}$ Diabetes Unit, Massachusetts General Hospital, Harvard Medical School, Boston, MA

${ }^{11}$ Department of Medicine, Harvard Medical School, Boston, MA

${ }^{12}$ Togo Ministry of Health, Lomé, Togo

${ }^{13}$ Caribbean Public Health Agency, Port of Spain, Trinidad and Tobago

${ }^{14}$ Division of Non-communicable Diseases, Kenya Ministry of Health, Nairobi, Kenya

${ }^{15}$ Health Research and Epidemiology Unit, Ministry of Health, Thimphu, Bhutan

${ }^{16}$ Department of Epidemiology and Biostatistics, School of Public Health, Makerere University, Kampala, Uganda

${ }^{17}$ Laboratory of Epidemiology of Chronic and Neurological Diseases, Faculty of Health Sciences, University of Abomey-Calavi, Cotonou, Benin 
of Health and Disease paradigm suggests that individuals exposed to adverse environmental influences, particularly malnutrition, in utero and in early childhood are predisposed to develop NCDs such as type 2 diabetes in adulthood (5). Possible mechanisms involved in the developmental programming of type 2 diabetes have been suggested to include structural and functional alterations affecting glucose metabolism, such as reduced $\beta$-cell mass and pancreatic insulin secretion (6).

Although adverse conditions in early life have commonly been assessed by body weight (7), they also translate into reduced attained adult height at the population level $(8,9)$. Undernutrition in utero and in early life as well as repeated or chronic infections restrict individuals from reaching their full physical growth potential and may cause low birth weight, early childhood stunting, and, ultimately, shorter attained adult height $(8,10)$. Height in high-income countries has been estimated to be $\sim 20 \%$ environmentally determined (11). In low-resource settings, in which food scarcity and infectious diseases are more frequent and more severe, the environmentally determined component of height appears to be higher (11), and it has been suggested that adult height can be restricted by $\geq 10-15 \mathrm{~cm}$ in the most extreme conditions (12). The extent to which variation in height among distinct populations is genetically determined is unclear. For example, in the U.S. and Europe, immigrants have tended to reach similar heights as those in the original populations within two generations, and advantaged groups in different populations tend to reach more similar

${ }^{18}$ Faculty of Agricultural and Food Sciences, American University of Beirut, Beirut, Lebanon

${ }^{19}$ National Institute for Medical Research, Dar es Salaam, Tanzania

${ }^{20}$ Nepal Health Research Council, Kathmandu, Nepal

${ }^{21}$ Faculty of Medicine and Health Sciences, Stellenbosch University, Stellenbosch, South Africa

${ }^{22}$ Faculty of Medicine and Health Sciences, National University of East Timor, Dili, Timor-Leste ${ }^{23}$ Ministry of Health, Solidarity, Social Cohesion and Gender, Government of the Union of Comoros, Moroni, Union of Comoros

${ }^{24}$ National Center for Public Health, Ulaanbaatar, Mongolia

${ }^{25}$ Department of Epidemiology and Population Health, Faculty of Health Sciences, American University of Beirut, Beirut, Lebanon

${ }^{26}$ Non-Communicable Disease Department, $\mathrm{Na}$ tional Center for Disease Control and Public Health, Tbilisi, Georgia height than is observed between different social groups within populations $(13,14)$.

Adult height relates to commonly accepted risk factors for diabetes, such as $\mathrm{BMI}$, in a number of ways. First, BMI might underestimate diabetes risk in populations in whom adverse conditions in early life are common (5). In light of the Developmental Origins of Health and Disease paradigm, relatively small increases in adiposity during adulthood might increase diabetes risk if individuals have been exposed to detrimental conditions in early life (15). Second, adult height predicts mortality independent of adiposity (16), and the relationship of height and several NCDs has been widely scrutinized. Adult height, for instance, is related to lower cardiovascular risk, but higher cancer risk (5). Third, in contrast to BMI, adult height is largely determined by late adolescence and is therefore potentially less vulnerable to reverse causation bias compared with anthropometric indicators that are more volatile over time. This may particularly be the case in cross-sectional studies in which the time ordering between the exposure and outcome (diabetes) cannot be established clearly.

Evidence on the relationship between adult height and diabetes is limited (5), but appears to point to an inverse association, consistent with the Developmental Origins of Health and Disease paradigm $(17,18)$. A recent systematic review and meta-analysis of 15 crosssectional studies and 10 cohort studies concluded that shorter height is associated with increased risk of type 2 diabetes (18). Intensified screening and prevention efforts in shorter individuals have been suggested (19), as well as the inclusion of height in the calculation of diabetes risk scores $(20,21)$. Existing evidence on the relationship between adult height and diabetes, however, is limited to mostly heterogeneous studies focused on high-income settings (18). While the Developmental Origins of Health and Disease paradigm is not restricted to certain populations, its effects may be more pronounced in contexts in which adverse environmental factors, such as fetal and early childhood malnutrition, are highly prevalent (3). Further, in rapidly developing countries, individuals who face nutritional deficiencies in early life may be met with abundance at later stages, making a detrimental metabolic adaptation a likely risk factor for chronic diseases such as type 2 diabetes in these settings (22). In this study, we therefore pooled individual-level, nationally representative survey data from $25 \mathrm{LMICs}$ ( $N=$ 554,122 ), including China and India. Our aim was to systematically assess the relationship between measured attained adult height and diabetes.

\section{RESEARCH DESIGN AND METHODS}

\section{Data Sources}

Prior to data acquisition, we defined several inclusion criteria for eligible surveys. Surveys were considered eligible if they: 1) were conducted later than 2004; 2) were nationally representative; 3) contained individual-level data; 4) had a response rate of at least $50 \%$; 5) assessed anthropometric measurements and diabetes biomarkers; 6) took place in a country considered a low-, lower-middle-,

\footnotetext{
${ }^{27}$ Eswatini Ministry of Health, Mbabane, Eswatin ${ }^{28}$ Liberia Ministry of Health, Monrovia, Liberia

${ }^{29}$ Medical Research Council/Wits Rural Public Health and Health Transition Research Unit, School of Public Health, University of the Witwatersrand, Johannesburg, South Africa

${ }^{30}$ Institute of Applied Health Research, University of Birmingham, Birmingham, U.K.

${ }^{31}$ Department of Global Health and Population, Harvard T.H. Chan School of Public Health, Boston, MA

${ }^{32}$ Department of Global Health and Social Medicine, Harvard Medical School, Boston, MA

${ }^{33}$ Harvard Center for Population and Development Studies, Cambridge, MA

${ }^{34}$ Department of Social and Behavioral Sciences, Harvard T.H. Chan School of Public Health, Boston, MA

${ }^{35}$ Africa Health Research Institute, Somkhele, South Africa
}

\begin{abstract}
${ }^{36}$ Public Health Foundation of India, New Delhi, India

Corresponding author: Jan-Walter De Neve, janwalter.deneve@uni-heidelberg.de

Received 4 January 2020 and accepted 11 June 2020

This article contains supplementary material online at https://doi.org/10.2337/figshare.12556205.

(C) 2020 by the American Diabetes Association. Readers may use this article as long as the work is properly cited, the use is educational and not for profit, and the work is not altered. More infor-

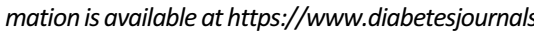
.org/content/license.
\end{abstract}


or upper-middle-income country according to the World Bank classification in the year the survey was conducted; and, lastly, 7) contained information on the educational attainment and wealth status of respondents. As a first step, we identified 109 existing World Health Organization (WHO) STEPwise approach to Surveillance (STEPS) NCD risk factor surveys, of which 49 fulfilled our inclusion criteria and 16 could eventually be acquired and included in our data set (23). These included Benin, Bhutan, Comoros, Eswatini, Georgia, Guyana, Kenya, Lebanon, Liberia, Mongolia, Nepal, Saint Vincent and the Grenadines (SVG), Tanzania, Timor-Leste, Togo, and Uganda (Supplementary Fig. 1). As a second step, we systematically searched for applicable non-STEPS health surveys. We were able to identify 97 further surveys, of which 21 fulfilled the inclusion criteria and 7 could be finally obtained and included (see Supplementary Box 1 for search algorithm). These included $\mathrm{La}$ Encuesta Nacional de Salud y Nutrición (Ecuador), the Demographic and Health Surveys (Bangladesh, India, and Namibia), the Mexico Family Life Survey, the Study for the Evaluation of Prevalence of Hypertension and Cardiovascular Risk (Romania), and, lastly, the South African National Health and Nutrition Examination Survey. We also included the China Health and Nutrition Survey, which represents substantial variation in geography, health indicators, and economic development in China, and the Indonesian Family Life Survey, which represents $83 \%$ of the Indonesian population (Supplementary Fig. 2). Our final data set included 25 surveys collected between 2008 and 2016. Country-specific sampling methods and sources are reported for each survey in Supplementary Table 1. Briefly, 13 of the surveys (Bangladesh, Comoros, Eswatini, Georgia, Guyana, India, Mexico, Namibia, Romania, SVG, Timor-Leste, Togo, and Uganda) used a two-staged random sampling process, while other surveys included further stages in their sampling structure. Twelve of the surveys stratified their sample, typically by urban and rural areas (Bangladesh, Comoros, India, Indonesia, Mongolia, Namibia, Nepal, Romania, South Africa, SVG, Timor-Leste, and Uganda).

\section{Study Population}

We included participants 25 to 64 years of age, as most STEPS surveys limit their biomarker assessment to this age range. We thus focus on type 2 diabetes and individuals who have not yet experienced a substantial loss in linear height due to aging (we examine alternative age criteria, however, in sensitivity analyses described below). We limited our analyses to participants with complete data on our exposure, outcome, and covariates. Average response rate, taking into account both household and individual response rates, was $88 \%$ across countries included in the study, yielding a final analytical sample of 554,122 individuals (Tables 1 and 2). Supplementary Fig. 3 displays a study participant flow diagram.

\section{Measurement of Exposure and End Points}

Our key exposure was attained adult height as a continuous variable in centimeters. For the WHO STEPS and Demographic and Health Surveys, which make up the majority of the surveys in our sample, adult height was typically measured once in a standing position using a portable height measuring board from Seca (24) or ShorrBoard (Weigh and Measure, LLC, Olney, MD) (25). Measurements were performed by trained staff following a detailed field manual. Individuals were defined as having diabetes based on WHO biomarker cutoffs: a fasting blood glucose of $\geq 7.0 \mathrm{mmol} / \mathrm{L}$; $a$ random blood glucose of $\geq 11.1 \mathrm{mmol} / \mathrm{L}$; or an $\mathrm{HbA}_{1 \mathrm{c}}$ level of $\geq 6.5 \%$ (26). Individuals with self-reported use of diabetes medication but normal biomarker values were also defined as having diabetes.

In 4 countries, glucose concentrations were measured in venous blood and in 18 countries in capillary blood, of which 12 were already provided as plasma equivalents. For the six remaining countries, we multiplied capillary glucose values by 1.11 , based on prior evidence suggesting that plasma glucose levels are often underestimated by capillary glucose levels (27). Three countries exclusively measured $\mathrm{HbA}_{1 \mathrm{c}}$. Individuals with missing information on fasting status were assumed to be fasting because they were instructed to fast as part of the study protocol for all countries, except in the case of India, where random blood glucose was measured.

\section{Covariates}

We included age (continuous), educational attainment (level attained at the time of the survey), and a measure of household wealth (quintiles) as covariates to control for potential confounding in our analysis. We constructed a household wealth index based on four different measurements of household wealth (income categories, continuous income, income quintiles, or an asset index). The surveys used different methodologies for the computation of wealth indices. For surveys assessing various dwelling characteristics and household possessions, we created an asset index based upon a principal component analysis according to the standard approach of the Demographic and Health Surveys, from which we then derived unweighted wealth quintiles. Countries that assessed wealth by self-reported household income mostly adhered to the WHO STEPS standard questionnaire. Participants were asked about average household income per week, month, or year in the past year and, if the question remained unanswered, had the possibility to choose the most applicable of several precoded income ranges. In accordance with the procedure by Harttgen and Vollmer (28), we used both the precoded ranges and the continuous income levels to again create unweighted wealth quintiles, assuming that national incomes follow a log-normal distribution. Additional details are provided elsewhere (29). In descriptive statistics, BMI was grouped into thin $\left(B M I<18.5 \mathrm{~kg} / \mathrm{m}^{2}\right.$ ), lower-normal (BMI $18.5-19.9 \mathrm{~kg} / \mathrm{m}^{2}$ ), normal (BMI 20.0$22.9 \mathrm{~kg} / \mathrm{m}^{2}$ ), upper-normal (BMI 23.0$24.9 \mathrm{~kg} / \mathrm{m}^{2}$ ), overweight (BMI 25-29.9 $\left.\mathrm{kg} / \mathrm{m}^{2}\right)$, and obesity $\left(\mathrm{BMI} \geq 30 \mathrm{~kg} / \mathrm{m}^{2}\right)$ (30).

\section{Statistical Analyses}

To determine the relationship between attained adult height and diabetes, we ran multilevel mixed-effects logistic regression models to obtain odds ratios (ORs) with 95\% Cls, controlling for potential confounders. To account for the hierarchical structure of our data set, we included random intercepts for world region and country in pooled analyses. We adjusted SEs for clustering at the highest-level random intercept or at the primary sampling unit level when applicable. Two different multiple models were fitted. In model 1, we only included diabetes and height as variables. In model 2 , our main model, we added age, education categories, and wealth quintiles. 
Table 1-Study country characteristics by world region

\begin{tabular}{|c|c|c|c|c|c|c|c|c|}
\hline Country & $\begin{array}{l}\text { Survey } \\
\text { year }\end{array}$ & $\begin{array}{c}\text { Response } \\
\text { rate, } \%\end{array}$ & $\begin{array}{l}\text { Sample } \\
\text { size }\end{array}$ & $\begin{array}{l}\text { Mean age, years } \\
\text { (weighted) }\end{array}$ & $\begin{array}{l}\text { Female, \% } \\
\text { (weighted) }\end{array}$ & Income group & $\begin{array}{c}\text { Stunted, } \\
\% \\
\end{array}$ & $\begin{array}{c}2015 \text { population, } \\
\text { thousands }\end{array}$ \\
\hline \multicolumn{9}{|c|}{$\begin{array}{l}\text { Latin America and } \\
\text { the Caribbean }\end{array}$} \\
\hline Ecuador & 2012 & 81.5 & 8,939 & 39.0 & 56.4 & Upper-middle & 25.4 & 16,144 \\
\hline Guyana & 2016 & 66.7 & 552 & 41.7 & 52.2 & Upper-middle & 11.3 & 769 \\
\hline Mexico & 2009-12 & 90.0 & 2,214 & 47.7 & 44.5 & Upper-middle & 13.6 & 125,891 \\
\hline SVG & 2013 & 67.8 & 631 & 41.9 & 56.4 & Upper-middle & - & 109 \\
\hline \multicolumn{9}{|c|}{$\begin{array}{l}\text { Eastern Europe and } \\
\text { the Middle East }\end{array}$} \\
\hline Georgia & 2016 & 75.7 & 2,018 & 44.6 & 53.4 & Lower-middle & 11.3 & 3,952 \\
\hline Lebanon & 2008-09 & 62.0 & 178 & 39.0 & 58.3 & Upper-middle & 16.5 & 5,851 \\
\hline Romania & 2015-16 & 69.1 & 1,253 & 44.2 & 53.4 & Upper-middle & 12.8 & 19,877 \\
\hline \multicolumn{9}{|c|}{$\begin{array}{l}\text { South, East, and } \\
\text { Southeast Asia }\end{array}$} \\
\hline Bangladesh & 2011 & 95.0 & 5,922 & 46.7 & 51.1 & Lower-middle & 41.3 & 161,201 \\
\hline Bhutan & 2014 & 96.9 & 2,240 & 39.1 & 41.5 & Lower-middle & 33.5 & 787 \\
\hline China & 2009 & 88.1 & 6,198 & 47.3 & 53.3 & Upper-middle & 9.0 & $1,397,029$ \\
\hline India & 2015-16 & 96.0 & 490,532 & 37.0 & 46.6 & Lower-middle & 37.9 & $1,309,054$ \\
\hline Indonesia & 2014 & 83.0 & 4,283 & 42.2 & 51.3 & Lower-middle & 36.4 & 258,162 \\
\hline Mongolia & 2009 & 95.0 & 1,111 & 39.6 & 45.2 & Lower-middle & 15.5 & 2,977 \\
\hline Nepal & 2013 & 98.6 & 3,046 & 39.8 & 52.7 & Low & 37.5 & 28,656 \\
\hline Timor-Leste & 2014 & 96.3 & 1,361 & 41.8 & 55.2 & Lower-middle & 50.9 & 1,241 \\
\hline \multicolumn{9}{|c|}{ Sub-Saharan Africa } \\
\hline Benin & 2008 & 99.0 & 3,228 & 43.5 & 50.5 & Low & 43.4 & 10,576 \\
\hline Comoros & 2011 & 96.5 & 1,227 & 40.9 & 74.8 & Low & 31.1 & 777 \\
\hline Eswatini & 2014 & 81.8 & 1,207 & 39.1 & 52.8 & Lower-middle & 25.5 & 1,319 \\
\hline Kenya & 2015 & 95.0 & 3,121 & 38.2 & 50.3 & Lower-middle & 26.2 & 47,236 \\
\hline Liberia & 2011 & 87.1 & 1,379 & 38.5 & 54.2 & Low & 41.8 & 4,500 \\
\hline Namibia & 2013 & 96.9 & 3,200 & 46.8 & 60.2 & Upper-middle & 22.7 & 2,426 \\
\hline South Africa & 2012 & 92.6 & 2,154 & 40.7 & 52.3 & Upper-middle & 27.2 & 55,291 \\
\hline Tanzania & 2012 & 94.7 & 4,085 & 39.1 & 47.5 & Low & 37.1 & 53,880 \\
\hline Togo & 2010 & 91.0 & 2,043 & 38.9 & 50.1 & Low & 27.6 & 7,417 \\
\hline Uganda & 2014 & 99.0 & 2,000 & 39.1 & 54.2 & Low & 28.9 & 40,145 \\
\hline World (all data) & - & 87.8 & 554,122 & 42.5 & 50.2 & 一 & 27.7 & $3,555,267$ \\
\hline
\end{tabular}

Mean age and percentage female calculated using sampling weights. The prevalence of early childhood stunting (Stunted, \%) in the year closest to the survey was derived from the joint United Nations International Children's Fund/WHO/World Bank database. Stunting was defined as below - 2 SDs from the median of the WHO 2006 reference population in terms of height-for-age. 2015 population size estimates were derived from the 2017 World Bank World Population Prospects.

We used equal weights for each country so that every country equally contributes to the analysis regardless of country sample size (we examine alternative country sampling weights in sensitivity analyses). We examined the relationship at the global level, by major world region and country, in addition to stratifying all analyses by sex. Descriptive statistics were calculated using sample weights.

\section{Sensitivity Analyses}

We subjected our regression results to a wide range of robustness checks. First, we added quadratic and cubic terms in age to account for possible nonlinearities in the relationship between age and diabetes. Second, we expanded our definition of diabetes to include individuals with self-reported diabetes diagnosis as having diabetes. Third, although participants were instructed to fast, we excluded individuals who did not explicitly confirm their fasting status. Fourth, we modeled our outcome using a Poisson regression model to obtain risk ratios. Risk ratios are more intuitively interpretable and similar to ORs when the binary outcome is relatively uncommon. Fifth, we used alternative specifications of our outcome including a continuous outcome (blood glucose or $\mathrm{HbA}_{1 \mathrm{c}}$ ), dysglycemia (fasting blood glucose of $\geq 5.6$ $\mathrm{mmol} / \mathrm{L}$ or $\mathrm{HbA}_{1 \mathrm{c}}$ level of $\geq 5.7 \%$ ), and diabetes diagnostic categories in multinomial regression models (normal, prediabetes, and diabetes) (31). Sixth, we used an alternative specification of our sample (aged $20-70$ years). Seventh, we converted our exposure for height into a categorical variable (quartiles) instead of a continuous variable. Eighth, we weighted countries proportional to their population size. We also conducted a "leave-one-out analysis" by rerunning our pooled analyses but excluding India. Ninth, although data on covariates were missing for relatively few respondents (2.2\%), we reran our main analyses after multiple imputation of missing variables in the data set. Tenth, we standardized height by conducting a $z$-transformation. We also used sex-specific WHO reference values for attained height at 19 years of age to externally standardize our exposure variable (32). Eleventh, we controlled for the diabetes ascertainment approach at the individual level to account for variation in diagnostic diabetes ascertainment (blood glucose, $\mathrm{HbA}_{1 \mathrm{c}}$, or 
Table 2-Selected characteristics of study participants

\begin{tabular}{|c|c|c|c|c|c|c|}
\hline \multirow[b]{2}{*}{ Characteristics } & \multicolumn{2}{|c|}{ Overall } & \multicolumn{2}{|c|}{ With diabetes } & \multicolumn{2}{|c|}{ Without diabetes } \\
\hline & $\begin{array}{c}\text { Unweighted } \\
N\end{array}$ & $\begin{array}{l}\text { Weighted mean or } \\
\text { percentage }\end{array}$ & $\begin{array}{c}\text { Unweighted } \\
n\end{array}$ & $\begin{array}{l}\text { Weighted mean or } \\
\text { percentage }\end{array}$ & $\begin{array}{c}\text { Unweighted } \\
n\end{array}$ & $\begin{array}{c}\text { Weighted mean or } \\
\text { percentage }\end{array}$ \\
\hline Height & 554,122 & 161.7 & 22,497 & 161.6 & 531,625 & 161.7 \\
\hline Age & 554,122 & 41.5 & 22,497 & 47.6 & 531,625 & 41.0 \\
\hline \multicolumn{7}{|l|}{ Sex } \\
\hline Male & 99,698 & 47.3 & 5,711 & 47.44 & 93,987 & 47.26 \\
\hline Female & 454,424 & 52.7 & 16,786 & 52.56 & 437,638 & 52.74 \\
\hline \multicolumn{7}{|l|}{ Education } \\
\hline None & 185,788 & 19.2 & 6,030 & 12.36 & 179,758 & 19.77 \\
\hline Primary & 92,201 & 28.5 & 4,187 & 32.63 & 88,014 & 28.14 \\
\hline Secondary or more & 276,133 & 52.3 & 12,280 & 55.01 & 263,853 & 52.09 \\
\hline \multicolumn{7}{|l|}{ Wealth quintile } \\
\hline Poorest & 103,555 & 19.3 & 2,596 & 17.30 & 100,959 & 19.46 \\
\hline Poorer & 113,735 & 19.8 & 3,278 & 20.87 & 110,457 & 19.67 \\
\hline Middle & 114,933 & 19.2 & 4,183 & 14.94 & 110,750 & 19.54 \\
\hline Richer & 111,735 & 20.5 & 5,703 & 23.22 & 106,032 & 20.28 \\
\hline Richest & 110,164 & 21.2 & 6,737 & 23.66 & 103,427 & 21.05 \\
\hline \multicolumn{7}{|l|}{ BMI $\left(\mathrm{kg} / \mathrm{m}^{2}\right)$} \\
\hline Thin $(<18.5)$ & 82,623 & 7.14 & 1,483 & 2.93 & 81,140 & 7.48 \\
\hline Lower-normal (18.5-19.9) & 70,803 & 8.53 & 1,351 & 3.65 & 69,452 & 8.93 \\
\hline Normal (20.0-22.9) & 165,036 & 24.06 & 4,315 & 13.15 & 160,721 & 24.95 \\
\hline Upper-normal (23-24.9) & 88,185 & 15.88 & 3,497 & 11.91 & 84,688 & 16.21 \\
\hline Overweight (25-29.9) & 110,015 & 26.62 & 7,441 & 32.03 & 102,574 & 26.17 \\
\hline Obesity ( $\geq 30$ ) & 37,460 & 17.77 & 4,410 & 36.32 & 33,050 & 16.26 \\
\hline
\end{tabular}

Mean or percentage use sample weights provided by the individual surveys and rescaled so that every country contributes equally.

both). Lastly, we added BMI (continuous) as a possible mediator in the relationship between adult height and diabetes.

\section{Heterogeneity}

We explored heterogeneity in the relationship between attained adult height and diabetes by key individual characteristics (educational attainment and birth cohort). The association between attained adult height and diabetes, for instance, may vary between individuals who are born during affluent, normal, or poor periods $(10,13)$. In the absence of Iongitudinal or time-series data, we therefore stratified our analyses by birth cohort characteristics (infant mortality rate). We also stratified our analyses by changes in living standards over an individual's life course (4), using the absolute decrease in infant mortality rate between infancy and adulthood. In doing so, we hypothesized that those who experienced substantial changes in living standards over time may have been at particular increased risk of developing diabetes later in life, because the early life adaptive response to adversity may be detrimental when the nutritional environment improves drastically later on in life (22). Additional details on these analyses are presented in Supplementary Text 1 in the Supplementary Material.

\section{Data Availability and Ethics}

This study includes individual-level data with measured height and biomarkers for diabetes from 25 countries. Some of these data are publicly available, while other data are available by request to the country (e.g., the WHO STEPS surveys). We have arranged specific data use agreements for surveys that are not publicly accessible and are therefore unable to share these data given the terms of our agreements. Supplementary Table 1 provides a complete list of survey reports with country contacts through whom those data that are not publicly available may be requested. Stata version 15.1 was used for all statistical analyses. This study was approved by the Harvard T.H. Chan School of Public Health Institutional Review Board (Boston, MA).

\section{RESULTS}

\section{Sample Description}

Our final sample included 554,122 individuals across 25 LMICs. In our study population, attained adult height was on average $161.7 \mathrm{~cm}$ (95\% Cl 161.2-162.3). This estimate is $\sim 8 \mathrm{~cm}$ shorter than the average in the U.S. (a substantial difference considering that height typically has an SD of $\sim 7 \mathrm{~cm}$ ) (33). Overall, crude prevalence of diabetes in our sample was 7.5\% (95\% Cl 6.9-8.2) (Table 2).

\section{Association Between Adult Height and Diabetes in LMICs}

Regression models using pooled individuallevel data from 25 LMICs show that, contrary to expectations based on the Developmental Origins of Health and Disease paradigm ( 3 ) and prior evidence $(17,18)$, the ORs of height and diabetes fluctuated closely around 1.0, mostly without reaching conventional benchmarks of statistical significance (Fig. 1 and Supplementary Table 2). As a notable exception, when stratifying our models by world region, we found that men in South, East, and Southeast Asia who were $1 \mathrm{~cm}$ taller had 3.1\% higher odds (95\% Cl $0.6-5.6)$ of having diabetes (as opposed to lower as hypothesized). Results were consistent across a wide range of sensitivity analyses, including when using alternative specifications of our outcome, covariates, sample, and sample weights (Supplementary Figs. 4-18 and Supplementary Tables 3-6).

In Fig. 2, we show regression results for the relationship between attained adult height and diabetes using individuallevel data stratified by country and sex. ORs were closely distributed around 1.0 


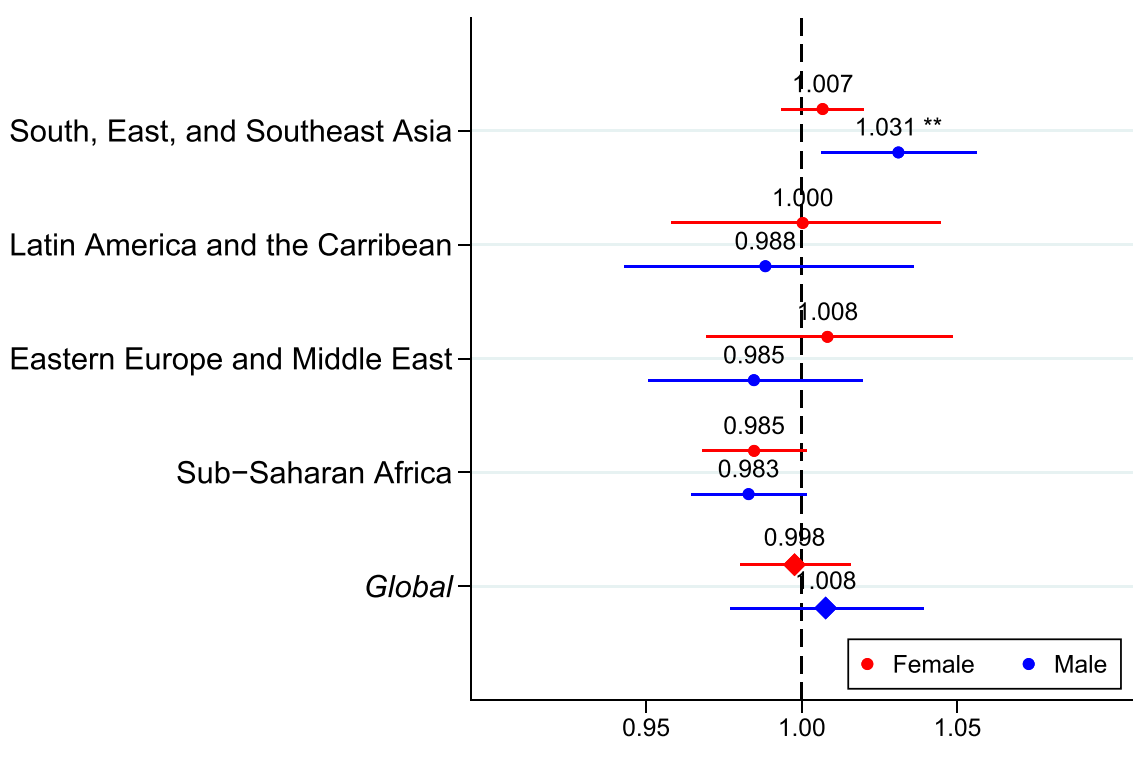

No Diabetes

Diabetes

Figure 1-Global- and world region-sex-stratified ORs of height and diabetes. Figure shows adjusted ORs from multivariable mixed-effects logistic regression models in the pooled sample and by world region, separately for women (red) and men (blue). The outcome was diabetes based on measured biomarkers, and exposure was measured height in centimeters. The OR reflects the change in odds with every centimeter gain in height. Globally, the OR of having diabetes fluctuated closely around 1.0. All models controlled for age (years), education, and household wealth and included a random intercept for country. Additionally, in the pooled analysis, a random intercept for world region was included. Countries were weighted equally. Full regression output and sensitivity analyses, including using alternative specifications of the model, variables, sample, and sample weights, are presented in Supplementary Figs. 4-18 and Supplementary Tables 2-6. Error bars represent $95 \% \mathrm{Cls} . N=554,122 .{ }^{* *} P<0.05$ (two-sided).

and did not reach statistical significance with a few exceptions. The previously reported inverse relationship between attained adult height and diabetes was found in $5 \%$ of analyses ( 2 out of 50 ). In Namibia, for instance, women who were $1 \mathrm{~cm}$ taller had $3.3 \%$ lower odds $(95 \% \mathrm{Cl}$ 0.5-6.1) of having diabetes. We also identified positive associations between attained adult height and diabetes among women in China, Indonesia, and TimorLeste, as well as among men in Tanzania and Timor-Leste. In Indonesia, for instance, women who were $1 \mathrm{~cm}$ taller had $4.5 \%$ higher odds $(95 \% \mathrm{Cl} 2.5-6.6)$ of having diabetes.

\section{Heterogeneity}

In our main analyses, we examined the relationship between attained adult height and diabetes at the global level, by major world region, individual country, and sex. In Supplementary Figs. 19-21, we show results for heterogeneity in the relationship between adult height and diabetes by additional individual characteristics (educational attainment and birth cohort).
Consistent with our main findings, ORs for diabetes were generally closely distributed around 1.0. When stratifying our analyses by educational attainment, none of the coefficients on adult height reached conventional significance levels (Supplementary Fig. 19). When stratifying our analyses by birth cohort-level infant mortality rate, the relationship between adult height and diabetes appeared somewhat more pronounced among men who were born during periods with low infant mortality rates (as opposed to high mortality rates as hypothesized) (Supplementary Fig. 20).

\section{CONCLUSIONS}

Using nationally representative survey data from 554,122 individuals, we found little evidence for a relationship between measured attained adult height and diabetes across 25 LMICs globally or in most world regions. When stratifying our analysis by country and sex, we identified the previously described inverse association between adult height and diabetes in $5 \%$ of analyses. Our results were consistent across a wide array of robustness checks, including when using alternative specifications of our model, exposure, outcome, analytical sample, and sample weights. Our findings suggest that adult height may play a more muted role in diabetes in LMICs than previously suggested by studies mostly focused on high-income country settings (17-20). To our knowledge, no other study has analyzed biomarker data on this scale to further our understanding of the link between attained adult height and diabetes in low-income and middle-income settings.

This study has several implications. First, our findings imply that the aspects of fetal and childhood nutrition influencing adult height likely differ from those predisposing individuals for adiposity and diabetes. Second, our findings point to a potential "decoupling" of risk factors for chronic undernutrition and diabetes, possibly resulting from the emergence of other different underlying drivers. Although undernutrition and obesity are both thought to be caused by poor-quality diets (such as low-quality calories) (34), their determinants are likely to be different if chronic undernutrition (as proxied by adult height) and diabetes were not associated. Third, as we found no relationship between height and diabetes in LMIC settings where adverse environmental conditions are highly prevalent, it appears unlikely that these conditions explain inverse associations observed in populations in high-income countries. The findings challenge prior evidence from more affluent settings, which has interpreted an inverse association between adult height and diabetes on the basis of the Developmental Origins of Health and Disease paradigm $(17,18)$. Based on evidence from high-income countries, intensified screening and prevention efforts in shorter individuals have recently been suggested (19), as well as the inclusion of adult height in the calculation of diabetes risk scores $(20,21)$. Policy makers in LMICs and funders of international development programs, however, should be aware of the lack of association between adult height and diabetes when designing policies aimed at reducing chronic disease in developing settings.

Despite our large-scale data collection effort to obtain both measured height and biomarker data on diabetes globally, this study has several limitations. First, 

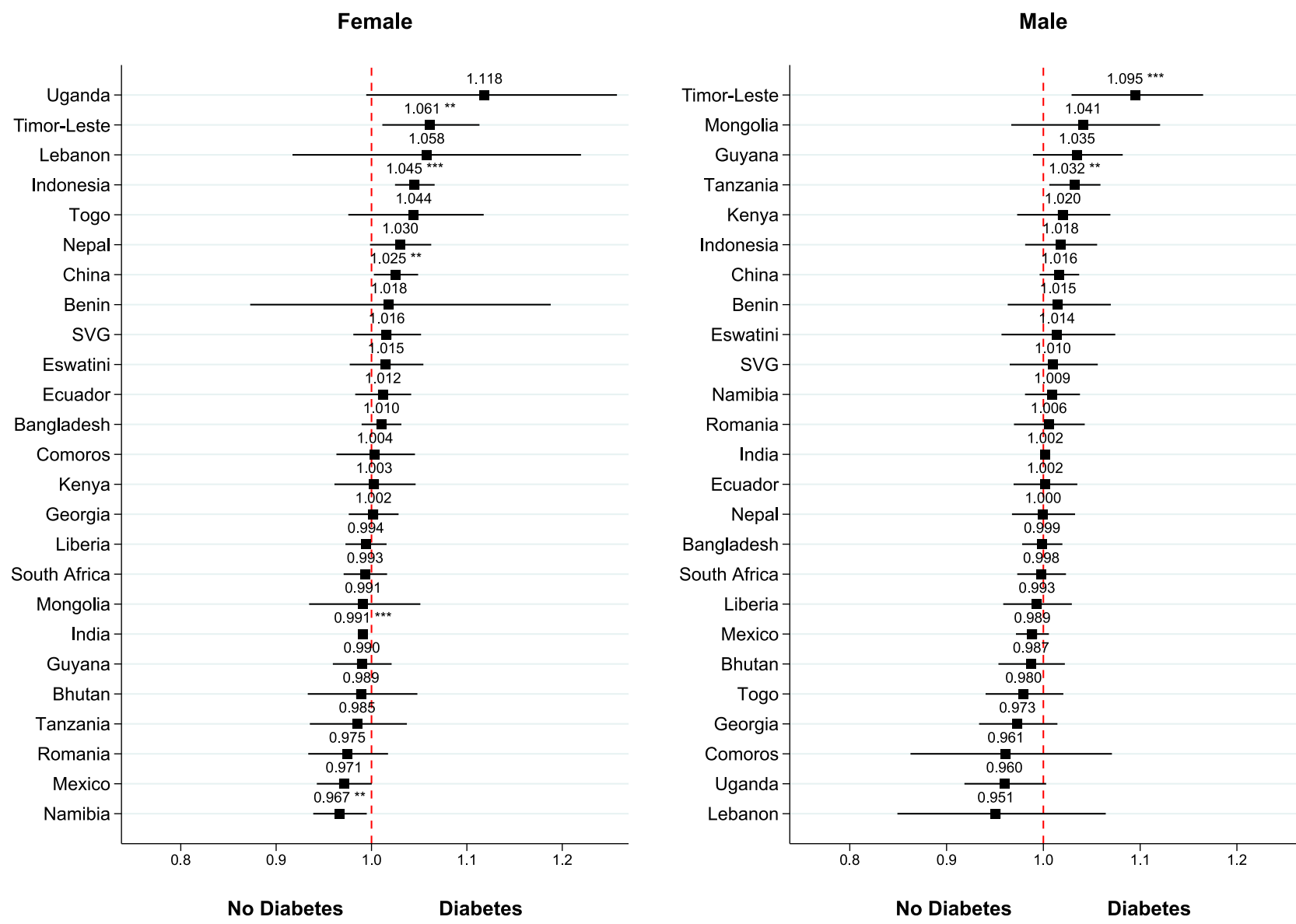

Figure 2-Country-sex-stratified ORs of height and diabetes. Figure shows adjusted ORs from multivariable logistic regression models by country, separately for women (left panel) and men (right panel). The outcome was diabetes based on measured biomarkers, and exposure was height measured in centimeters. The OR reflects the change in odds with every centimeter gain in height. The ORs of having diabetes at the sex-country level were closely distributed around 1.0. All models controlled for age (years), education, and household wealth. Sensitivity analyses, including using alternative specifications of the model, variables, sample, as well as sample weights, are presented in Supplementary Figs. 4-18 and Supplementary Tables 3-6. Error bars represent $95 \%$ Cls. $N=554,122$. ${ }^{* *} P<0.05 ; * * * P<0.01$ (two-sided).

although we controlled for known confounders, our study design does not allow testing for causal inference. Second, diabetes measurements may have yielded inaccuracies as biomarkers were generally measured only once, in capillary instead of venous blood, and fasting status was verified by self-report. Nonetheless, a key strength of our study was the availability of these individual-level biomarker data for diabetes as opposed to examining risk factors of diabetes as the primary outcome, such as BMI (35). Third, we were not able to distinguish between type 1 and type 2 diabetes. However, the incidence of type 1 diabetes is relatively low compared with type 2 diabetes, particularly in the age group $>25$ years of age (36). Fourth, our results may not generalize beyond the LMICs analyzed in the current study. Fifth, to interpret the association of attained adult height and diabetes in the context of the Developmental Origins of Health and Disease paradigm, one must rely on the assumption that adult height is a plausible proxy for environmental conditions in early life, particularly malnutrition, at the population level (37). Adults, however, may be of shorter height for reasons unrelated to childhood living conditions (8). Individuals with exposure to childhood stunting and catch-up growth can also not be differentiated from individuals with normal growth. Nevertheless, there is only a narrow time frame in which complete catch-up growth is possible $(38,39)$.

Furthermore, observed adult height may conceal other important consequences of adverse exposures in early life. Specifically, adversity in early life may affect observed adult height at the population level through two main channels: scarring and selective mortality. Scarring occurs when the entire distribution of height is shifted downward due to an adverse exposure in early life (40). Selective mortality, in contrast, can bias downward, cancel out, or even dominate over the scarring effects, resulting in greater observed height in adulthood due to mortality being higher among children with underlying health problems. Selective mortality has been suggested to dominate over scarring when conditions are particularly harsh, such as in the Great Chinese famine and the Great Irish famine, as well as in a number of sub-Saharan African countries, such as Chad and Mali (13). Nevertheless, attained adult height has been shown to be a measure of cumulative net nutrition on a population level (8).

As a conclusion, adult height is not associated with diabetes across LMICs, in contrast to findings from high-income country settings. Environmental factors in early life reflected in attained adult height 
likely differ from those predisposing individuals for diabetes.

Acknowledgments. The authors thank each of the country-level survey teams and study participants who made this analysis possible.

Funding. F.T. was supported by the Else KrönerFresenius-Stiftung within the Heidelberg Graduate School of Global Health. P.G. was supported by the National Center for Advancing Translational Sciences of the National Institutes of Health (KL2TR003143). T.B. was supported by the Alexander von Humboldt Foundation through the Alexander von Humboldt Professor award, funded by Germany's Federal Ministry of Education and Research. J.-W.D.N. was supported by the Alexander von Humboldt Foundation, funded by Germany's Federal Ministry of Education and Research, Deutsche Forschungsgemeinschaft (405898232), the Eunice Kennedy Shriver National Institute of Child Health and Human Development of the National Institutes of Health (R03-HD-098982), and the Heidelberg University Excellence Initiative. This article was also part of research funded under the European Union's Research and Innovation program Horizon 2020 (project 825823). Duality of Interest. No potential conflicts of interest relevant to this article were reported. Author Contributions. F.T., T.B., and J.-W.D.N. conceived and designed the study. P.G., J.M.-G. and L.M.J. supervised data collation. F.T. performed the statistical analysis under the supervision of P.G. J.M.-G., T.B. and J.-W.D.N. F.T. and J.-W.D.N. wrote the first draft of the manuscript. All authors made important revisions to the manuscript. All authors read and approved the final manuscript. J.-W.D.N. is the guarantor of this work and, as such, had full access to all of the data in the study and takes responsibility for the integrity of the data and the accuracy of the data analysis.

Prior Presentation. This study was presented in an asynchronous oral session at the Virtual Annual Meeting of the Population Association of America, 22-25 April 2020.

\section{References}

1. Danaei G, Finucane MM, Lu Y, et al.; Global Burden of Metabolic Risk Factors of Chronic Diseases Collaborating Group (Blood Glucose). National, regional, and global trends in fasting plasma glucose and diabetes prevalence since 1980: systematic analysis of health examination surveys and epidemiological studies with 370 country-years and 2.7 million participants. Lancet 2011:378:31-40

2. Roth GA, Abate $D$, Abate KH, et al.; GBD 2017 Causes of Death Collaborators. Global, regional, and national age-sex-specific mortality for 282 causes of death in 195 countries and territories, 1980-2017: a systematic analysis for the Global Burden of Disease Study 2017. Lancet 2018;392 1736-1788

3. Davies JI, Macnab AJ, Byass P, et al. Developmental origins of health and disease in Africainfluencing early life. Lancet Glob Health 2018; 6:e244-e245

4. Hales CN, Barker DJ. Type 2 (non-insulindependent) diabetes mellitus: the thrifty phenotype hypothesis. Diabetologia 1992;35:595-601
5. Stefan N, Häring $\mathrm{HU}, \mathrm{Hu} F B$, Schulze $M B$. Divergent associations of height with cardiometabolic disease and cancer: epidemiology, pathophysiology, and global implications. Lancet Diabetes Endocrinol 2016;4:457-467

6. Warner MJ, Ozanne SE. Mechanisms involved in the developmental programming of adulthood disease. Biochem J 2010;427:333-347

7. Whincup PH, Kaye SJ, Owen CG, et al. Birth weight and risk of type 2 diabetes: a systematic review. JAMA 2008;300:2886-2897

8. Perkins JM, Subramanian SV, Davey Smith G, Özaltin E. Adult height, nutrition, and population health. Nutr Rev 2016;74:149-165

9. NCD Risk Factor Collaboration (NCD-RisC). A century of trends in adult human height. elife 2016;5:e13410

10. Bozzoli C, Deaton A, Quintana-Domeque C. Adult height and childhood disease. Demography 2009;46:647-669

11. Silventoinen $\mathrm{K}$. Determinants of variation in adult body height. J Biosoc Sci 2003;35:263-285 12. Steckel RH. Heights and human welfare: recent developments and new directions. Explor Econ Hist 2009;46:1-23

13. Deaton A. Height, health, and development Proc Natl Acad Sci U S A 2007;104:13232-13237 14. Falkner F, Tanner JM. Methodology and ecological, genetic, and nutritional effects on growth. In Human Growth: A Comprehensive Treatise. Falkner F, Tanner JM, Eds. New York, Plenum Press, 1986, p. 241-262

15. Lumey LH, Khalangot MD, Vaiserman AM. Association between type 2 diabetes and prenatal exposure to the Ukraine famine of 1932-33 a retrospective cohort study. Lancet Diabetes Endocrinol 2015;3:787-794

16. Emerging Risk Factors Collaboration. Adult height and the risk of cause-specific death and vascular morbidity in 1 million people: individual participant meta-analysis. Int J Epidemiol 2012 41:1419-1433

17. Janghorbani M, Momeni F, Dehghani M. Hip circumference, height and risk of type 2 diabetes: systematic review and meta-analysis. Obes Rev 2012;13:1172-1181

18. Shrestha S, Rasmussen SH, Pottegård A, et al. Associations between adult height and type 2 diabetes mellitus: a systematic review and metaanalysis of observational studies. J Epidemiol Community Health 2019;73:681-688

19. Wittenbecher $\mathrm{C}$, Kuxhaus $\mathrm{O}$, Boeing $\mathrm{H}$ Stefan N, Schulze MB. Associations of short stature and components of height with incidence of type 2 diabetes: mediating effects of cardiometabolic risk factors. Diabetologia 2019;62:2211-2221

20. Schulze MB, Hoffmann $\mathrm{K}$, Boeing $\mathrm{H}$, et al. An accurate risk score based on anthropometric, dietary, and lifestyle factors to predict the development of type 2 diabetes. Diabetes Care 2007:30:510-515

21. Mühlenbruch K, Ludwig T, Jeppesen $C$, et al. Update of the German Diabetes Risk Score and external validation in the German MONICA/KORA study. Diabetes Res Clin Pract 2014;104:459466

22. Robinson $R$. The fetal origins of adult disease. BMJ 2001:322:375-376

23. Riley L, Guthold R, Cowan M, et al. The World Health Organization STEPwise approach to noncommunicable disease risk-factor surveillance: methods, challenges, and opportunities. Am J Public Health 2016;106:74-78

24. World Health Organization. WHO STEPS Surveillance Manual. Geneva, Switzerland, World Health Organization, 2017

25. The Nambia Ministry of Health and Social Services (MoHSS) and ICF International. The Namibia Demographic and Health Survey 2013. Demographic and health surveys. Windhoek, Namibia, and Rockville, MA, The Nambia Ministry of Health and Social Services (MoHSS) and ICF International, 2014

26. World Health Organization (WHO). Definition and Diagnosis of Diabetes Mellitus and Intermediate Hyperglycemia: Report of a WHO/ IDF Consultation. Geneva, Switzerland, World Health Organization, 2006

27. Sacks DB, Arnold M, Bakris GL, et al. Guidelines and recommendations for laboratory analysis in the diagnosis and management of diabetes mellitus. Clin Chem 2011;57:e1-e47

28. Harttgen $\mathrm{K}$, Vollmer S. Using an asset index to simulate household income. Econ Lett 2013;121: 257-262

29. Seiglie JA, Marcus ME, Ebert C, et al. Diabetes prevalence and its relationship with education, wealth, and BMI in 29 low- and middle-income countries. Diabetes Care 2020;43:767-775

30. WHO Expert Consultation. Appropriate bodymass index for Asian populations and its implications for policy and intervention strategies. Lancet 2004;363:157-163

31. American Diabetes Association. (2) Classification and diagnosis of diabetes. Diabetes Care 2015;38(Suppl.):S8-S16

32. de Onis M. WHO Child Growth Standards. Length/Height-for-Age, Weight-for-Age, Weightfor-Length, Weight-for-Height and Body Mass Index-for-Age. Methods and Development. Geneva, Switzerland, World Health Organization, 2006

33. Fryar $C D, G u$ Q, Ogden $C L$, Flegal KM. Anthropometric reference data for children and adults: United States, 2011-2014. Vital Health Stat 3 2016;39:1-46

34. Swinburn BA, Kraak VI, Allender S, et al. The global syndemic of obesity, undernutrition, and climate change: the Lancet Commission report. Lancet 2019;393:791-846

35. Bixby H, Bentham J, Zhou B, et al.; NCD Risk Factor Collaboration (NCD-RisC). Rising rural bodymass index is the main driver of the global obesity epidemic in adults. Nature 2019;569:260-264

36. Diaz-Valencia $P A$, Bougnères $P$, Valleron $A-J$. Global epidemiology of type 1 diabetes in young adults and adults: a systematic review. BMC Public Health 2015;15:255

37. Koncz V, Geldsetzer P, Manne-Goehler J, et al. Shorter height is associated with diabetes in women but not in men: nationally representative evidence from Namibia. Obesity (Silver Spring) 2019;27:505-512

38. Victora CG, Adair L, Fall C, et al.; Maternal and Child Undernutrition Study Group. Maternal and child undernutrition: consequences for adult health and human capital. Lancet 2008;371:340-357 39. de Onis M, Branca F. Childhood stunting: a global perspective. Matern Child Nutr 2016; 12(Suppl. 1):12-26

40. Ozaltin E, Hill K, Subramanian SV. Association of maternal stature with offspring mortality, underweight, and stunting in low- to middleincome countries. JAMA 2010;303:1507-1516 